\title{
Imaging in Cardiac Surgery: Visualizing the Heart
}

\author{
Arnaud Van Linden ${ }^{1}$ Jörg Seeburger ${ }^{2}$ Thilo Noack ${ }^{2}$ Volkmar Falk ${ }^{3}$ Thomas Walther ${ }^{1}$
}

\author{
${ }^{1}$ Department of Cardiac Surgery, Kerckhoff Klinik, Bad Nauheim, \\ Germany \\ 2 Department of Cardiac Surgery, Heart Center, Leipzig, Germany \\ ${ }^{3}$ Department of Cardiac Surgery, German Heart Center, Berlin, \\ Germany
}

Thorac Cardiovasc Surg 2017;65:S213-S216.

\section{Introduction}

In the physiology of human handling, the hand always follows the eye. Hence, optimal vision is indispensable to perform surgery. With the advent of minimally invasive cardiac surgery, the unobscured direct vision was no longer possible, and therefore endoscopy was introduced to enable it. Advanced endoscopic techniques, as well as catheterbased interventions, demanded improved preoperative planning based on echocardiographic or computed tomographic (CT) data. Therefore, cardiac surgeons had not only to understand and interpret imaging data but also integrate them into their daily practice. Prof. Mohr was one of the pioneers in the field as very early he realized the importance of imaging in the curriculum of a cardiac surgeon, and promoted the acquisition of imaging skills during the residency program in Leipzig. Consequently, several surgeons and anesthesiologists actively developed research projects in the field of image-guided therapy.

\section{Early Days}

During his research fellowship at Cedars-Sinai Medical Center, Prof. Mohr started his experimental and clinical research in the field of thermal coronary angiography. ${ }^{1}$ This technique was developed for intraoperative noninvasive testing of anastomotic patency and coronary flow by visualizing small temperature differences between adjacent structures. A young research colleague of Prof. Mohr at that time was Volkmar Falk, who wrote his thesis on this specific topic and they continued working in this imaging field in the early Leipzig days to develop thermal coronary angiography as a reliable intraoperative visualization tool to assess graft patency. ${ }^{2-5}$ Besides the academic interest in cardiac imaging, Prof. Mohr established an echocardiography group within

received

March 1, 2017

accepted

March 1, 2017

Address for correspondence Arnaud Van Linden, MD, Department of Cardiac Surgery, Kerckhoff-Klinik, Benekestr. 2-8, 61231 Bad

Nauheim, Germany (e-mail: a.vanlinden@kerckhoff-klinik.de).

the surgical department, to perform routine echocardiographic examinations and to foster research related to image-guided surgery. In the mid-1990s an echocardiography laboratory run by cardiac surgeons was quite rare in Germany but served as a testimony to the vast interest of Prof Mohr with regards to imaging as an integral part of a surgical career.

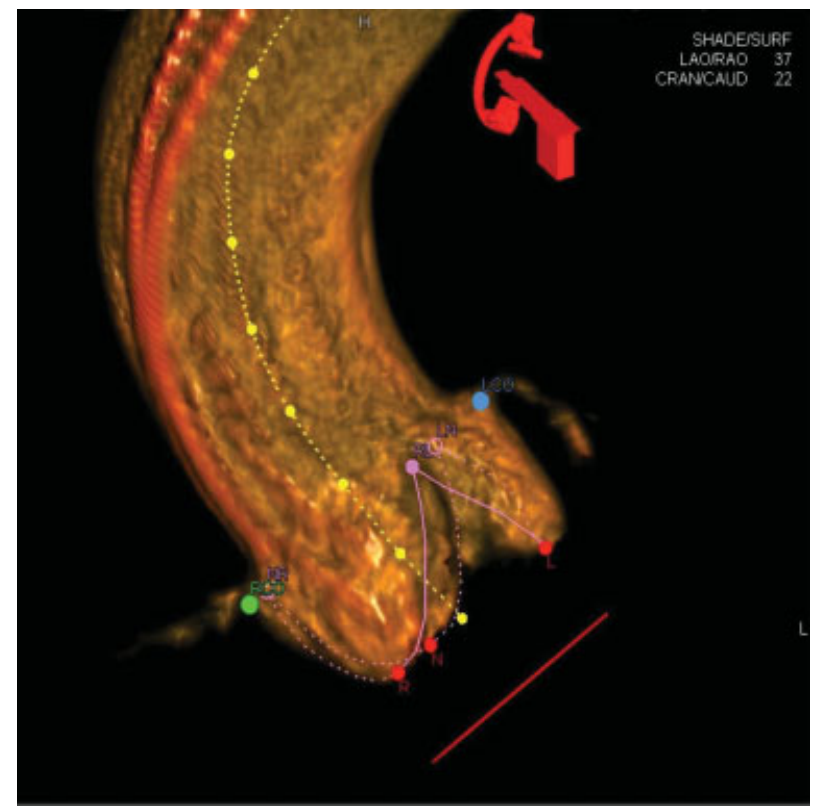

Fig. 1 Three-dimensional model of the aortic root generated by DynaCT: The C-arm angulation is presented in the right upper corner, the red line under the aortic annulus indicates a perpendicular angulation of the C-arm to the annulus, the green, and blue dots represent the right and left coronary ostia, the three red dots represent the three nadirs of the aortic valve, the yellow centerline is interpolated in the aorta, and the purple dots and lines indicate the commissures and their course.

(c) 2017 Georg Thieme Verlag KG Stuttgart · New York
DOI http://dx.doi.org/ 10.1055/s-0037-1601344. ISSN 0171-6425. 


\section{Expanding of Imaging to the Next Level}

In 2005, the "innovation center for computer assisted surgery (ICCAS)" was founded in collaboration with the Leipzig
Heart Center. One of the early foci of ICCAS was to develop the field of image-guided robotic surgery including augmented reality applications. ${ }^{6-8}$ This comprised planning, simulation, and performance of augmented reality enhanced
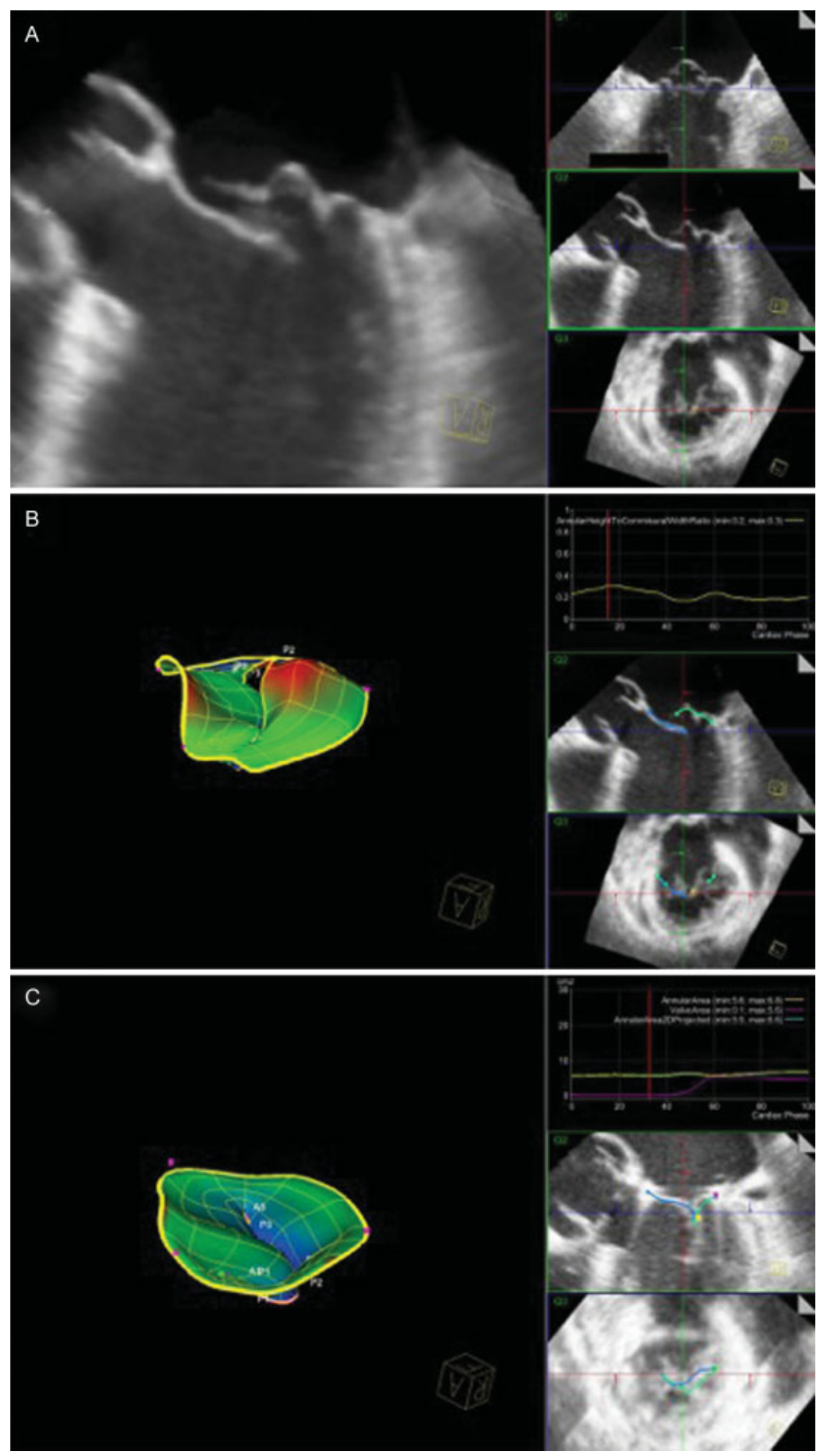

Fig. 2 MV assessment by four-dimensional quantification software. Three-dimensional transesophageal echocardiography image in mid-systole shows severe P2-prolapse (A). Assessment of MV morphology in mid-systole by MV model before surgical MV repair (B). Assessment of MV in midsystole after surgical MV repair. MV, mitral valve. 
robotically assisted endoscopic bypass grafting. Another augmented reality application was developed for determining the optimal annuloplasty ring size during mitral valve repair using a template overlay on top of three-dimensional transesophageal echocardiography (3D-TEE). ${ }^{9}$

\section{TAVI Imaging in Daily Cardiac Surgical Practice}

With the early introduction of transcatheter aortic valve implantation (TAVI) at the Leipzig Heart Center in 2005, ${ }^{10}$ a new demand for advanced imaging became obvious. Imaging was no longer only important for supporting intraoperative strategies but became indispensable to guide the procedure and to help to avoid life-threatening complications. The closed-heart situation of TAVI was a new setup for cardiac surgeons who were used to have direct vision of the operating field and the aortic valve. This led to the need for precise preoperative and intraoperative planning and sizing of the aortic root anatomy including the aortic annulus. Initially, TEE was the main imaging technique for preoperative annulus sizing. ${ }^{11,12}$ With growing experience in TAVI and the finding of a high percentage of paravalvular leakage the complex 3D geometry of the aortic annulus came into focus. ${ }^{13}$ First experiences with aortic annulus sizing in 3D-TEE showed precise measurements, ${ }^{14}$ but soon the potential advantage of $\mathrm{CT}$ for routine TAVI screening became evident. CT allows for a 3D view of all anatomic structures of interest, including calcification patterns. The Leipzig group adopted this technique quickly and presented the concept of the definition of an effective aortic annulus diameter based on the luminal circumference of the aortic annulus and the resulting calculated area. ${ }^{15}$ Ever since the effective diameter has become the standard now routinely used for CT-based annulus diameter measurements.

Intraoperative guidance and template-based planning are other important aspects of TAVI procedures. In close cooperation with ICCAS and Siemens Healthcare new concepts of intraoperative TAVI imaging were evaluated at the Leipzig Heart Center, in Zürich, Switzerland and in Bad Nauheim, Germany. Tracking of landmarks during live 2D fluoroscopy was developed as a tool to enable precise implantation of TAVI prostheses. In an ICCAS project, imaging-registration flowcharts were developed and integrated into fluoroscopy. ${ }^{16}$ The so-called "DynaCT," an intraoperative 200 degrees rotational angiography to generate 3D CT-like images (-Fig. 1) using a C-arm ${ }^{17,18}$ was for the first time applied to visualize the aortic root on top of the life fluoroscopy during TAVI procedures. This technique, which was also patented by the Leipzig team in conjunction with Siemens, was further developed clinically at the Leipzig Heart Center and the Kerckhoff Klinik in Bad Nauheim. DynaCT is now routinely used to optimize C-arm angulation. The latest generation of DynaCT software allows for annulus diameter and coronary ostia distance measurements. ${ }^{19}$ Early ideas of template-based overlay of prostheses models into the aortic root to predict implantation height and valve size ${ }^{20}$ were also integrated into this software.

\section{Advanced Mitral Valve Imaging}

The Leipzig Heart Center is well known for its minimally invasive mitral valve repair program. Advanced mitral valve imaging was always one of Prof. Mohr's major fields of interest. There was an early adoption of 3D-TEE for mitral valve surgery planning and judgment. ${ }^{8,21}$ Detailed anatomical and pathological, as well as a physiological understanding of the mitral valve apparatus, are indispensable for successful mitral valve surgery. Real-time 3D-TEE allows for examining all relevant structures preoperatively and intraoperatively and serves as a quality control postoperatively. Intraoperative TEE is also able to detect most of the severe complications that may occur during mitral valve surgery, such as iatrogenic injury of the circumflex coronary artery which was systematically explored in a large case series. ${ }^{22}$ The use of 3D-TEE soon became clinical routine in mitral valve surgery centers worldwide. Lately, augmented-reality applications, model base planning, and 4D modeling were clinical research areas at the Leipzig Heart Center. ${ }^{8,23,24}$ Different types of mitral valve models have been evaluated and established with different levels of complexity in calculating and understanding. Dynamic mitral valve models that can be reconstructed using 4D CT or real-time 3D-TEE have been found especially useful as they allow the evaluation of the mitral valve throughout the entire cardiac cycle. In the near future, structure-fluid interaction models will be explored. First experiences with 4D modeling of the mitral valve in real-time 3D-TEE (- Fig. 2 ) have been published ${ }^{23}$ and the clinical use in complex mitral valve repair planning has been demonstrated in a live case at 2016 EACTS annual meeting, held at Barcelona, Spain.

\section{References}

1 Mohr FW, Matloff J, Grundfest W, et al. Thermal coronary angiography: a method for assessing graft patency and coronary anatomy in coronary bypass surgery. Ann Thorac Surg 1989; 47(3):441-449

2 Falk V, Walther T, Kitzinger H, et al. An experimental approach to quantitative thermal coronary angiography. Thorac Cardiovasc Surg 1998;46(1):25-27

3 van Son JAM, Falk V, Walther T, Diegeler A, Mohr FW. Thermal coronary angiography for intraoperative testing of coronary patency in congenital heart defects. Ann Thorac Surg 1997; 64(5):1499-1500

4 Falk V, Diegeler A, Walther T, et al. Intraoperative patency control of arterial grafts in minimally invasive coronary artery bypass graft operations by means of endoscopic thermal coronary angiography. J Thorac Cardiovasc Surg 1997;114(3):507-509

5 Mohr FW, Falk V, Philippi A, et al. Intraoperative assessment of internal mammary artery bypass graft patency by thermal coronary angiography. Cardiovasc Surg 1994;2(6):703-710

6 Falk V, Mourgues F, Adhami L, et al. Cardio navigation: planning, simulation, and augmented reality in robotic assisted endoscopic bypass grafting. Ann Thorac Surg 2005;79(6):2040-2047

7 Falk V, Mourgues F, Vieville T, et al. Augmented reality for intraoperative guidance in endoscopic coronary artery bypass grafting. Surg Technol Int 2005;14:231-235

8 Ender J, Končar-Zeh J, Mukherjee C, et al. Value of augmented reality-enhanced transesophageal echocardiography (TEE) for 
determining optimal annuloplasty ring size during mitral valve repair. Ann Thorac Surg 2008;86(5):1473-1478

9 Ender J, Eibel S, Mukherjee C, et al. Prediction of the annuloplasty ring size in patients undergoing mitral valve repair using realtime three-dimensional transoesophageal echocardiography. Eur J Echocardiogr 2011;12(6):445-453

10 Walther T, Falk V, Borger MA, et al. Minimally invasive transapical beating heart aortic valve implantation-proof of concept. Eur J Cardiothorac Surg 2007;31(1):9-15

11 Walther T, Dewey T, Borger MA, et al. Transapical aortic valve implantation: step by step. Ann Thorac Surg 2009;87(1):276283

12 Moss RR, Ivens E, Pasupati S, et al. Role of echocardiography in percutaneous aortic valve implantation. JACC Cardiovasc Imaging 2008;1(1):15-24

13 Piazza N, de Jaegere P, Schultz C, Becker AE, Serruys PW, Anderson RH. Anatomy of the aortic valvar complex and its implications for transcatheter implantation of the aortic valve. Circ Cardiovasc Interv 2008;1(1):74-81

$14 \mathrm{Ng} \mathrm{AC}$, Delgado V, van der Kley F, et al. Comparison of aortic root dimensions and geometries before and after transcatheter aortic valve implantation by 2 - and 3-dimensional transesophageal echocardiography and multislice computed tomography. Circ Cardiovasc Imaging 2010;3(1):94-102

15 Kempfert J, Van Linden A, Lehmkuhl L, et al. Aortic annulus sizing: echocardiographic versus computed tomography derived measurements in comparison with direct surgical sizing. Eur J Cardiothorac Surg 2012;42(4):627-633

16 Merk DR, Karar ME, Chalopin C, et al. Image-guided transapical aortic valve implantation: sensorless tracking of stenotic valve landmarks in live fluoroscopic images. Innovations (Phila) 2011; 6(4):231-236

17 Kempfert J, Noettling A, John M, Rastan A, Mohr FW, Walther T. Automatically segmented DynaCT: enhanced imaging during transcatheter aortic valve implantation. J Am Coll Cardiol 2011; 58(25):e211

18 Van Linden A, Kempfert J, Nollert G, Walther T. Examining the use of imaging during T-AVI: Focus on online 3D DynaCT. Interv Cardiol 2012;4(3):361-370

19 Van Linden A, Kempfert J, Blumenstein J, et al. Manual versus automatic detection of aortic annulus plane in a computed tomography scan for transcatheter aortic valve implantation screening. Eur J Cardiothorac Surg 2014;46(2):207-212, discussion 212

20 Jacobs S, Gessat M, Walther T, Falk V. Three-dimensional template-based planning for transapical aortic valve implantation. J Thorac Cardiovasc Surg 2011;141(6):1541-1543

21 Fabricius AM, Walther T, Falk V, Mohr FW. Three-dimensional echocardiography for planning of mitral valve surgery: current applicability? Ann Thorac Surg 2004;78(2):575-578

22 Ender J, Selbach M, Borger MA, et al. Echocardiographic identification of iatrogenic injury of the circumflex artery during minimally invasive mitral valve repair. Ann Thorac Surg 2010;89(6): 1866-1872

23 Noack T, Mukherjee C, Kiefer P, et al. Four-dimensional modelling of the mitral valve by real-time 3D transoesophageal echocardiography: proof of concept. Interact Cardiovasc Thorac Surg 2015; 20(2):200-208

24 Noack T, Kiefer P, Ionasec R, et al. New concepts for mitral valve imaging. Ann Cardiothorac Surg 2013;2(6):787-795 